\title{
Desmoplastic Fibroma, Report of a Rare Case in Infraorbital Rim
}

\author{
Yaser Safi, Nafise Shamloo, ${ }^{2}$ Hossein Heidar, ${ }^{3}$ Solmaz Valizadeh, ${ }^{1}$ Mohammad Mehdi Agh- \\ dasi, ${ }^{1}$ and Maryam Eslami Manouchehri, ${ }^{1, *}$ \\ ${ }_{1}^{1}$ Department of Oral and Maxillofacial Radiology, School of Dentistry, Shahid Beheshti University of Medical Sciences, Tehran, Iran \\ ${ }^{2}$ Department of Oral and Maxillofacial Pathology, School of Dentistry, Shahid Beheshti University of Medical Sciences, Tehran, Iran \\ ${ }^{3}$ Department of Oral and Maxillofacial Surgery, Tehran University of Medical Sciences, Tehran, Iran \\ *Corresponding author: Maryam Eslami Manouchehri, Department of Oral and Maxillofacial Radiology, School of Dentistry, Shahid Beheshti University of Medical Sciences, Tehran, \\ Iran. Tel: +98-9126871870, E-mail: maryam_manouchehri@yahoo.com
}

Received: November 22, 2014; Revised: January 24, 2015; Accepted: February 16, 2015

\begin{abstract}
Desmoplastic fibroma (DF) is a rare and locally aggressive intraosseous tumor with unknown etiology. The mandible is the common site of involvement in the maxillofacial region. However, it is believed that DF can arise in any bone of the body. A wide age distribution has been reported for DF occurrence, extending from birth to the sixth decade of life, with a peak incidence at 10 to 19 years of age. In this study, diagnostic and therapeutic management of a 6-year-old girl with a desmoplastic fibroma of the inferior orbital rim and zygomatic buttress are discussed. Cone beam computed tomography (CBCT) revealed a mixed lesion in infraorbital rim, which had illdefined borders and a straight thick bony septum inside the lesion. It also involved the zygomatic process of maxilla and zygomatic bone. According to radiologic concepts, this rare lesion may mimic fibro-osseous, benign and especially malignant lesions. Regarding different treatment plans, identification of this lesion is essential. Furthermore, presence of coarse and irregular or straight septa along with some imaging criteria for malignant lesions such as destruction of the cortex, periosteal reaction and soft tissue invasion would be helpful to differentiate this lesion from malignant and multilocular benign lesions.
\end{abstract}

Keywords: Desmoplastic Fibroma; Zygoma; Bone Neoplasm

\section{Introduction}

Desmoplastic fibroma (DF) is a rare, benign and meanwhile locally aggressive intraosseous neoplasm (1-3), which commonly occurs in the first two decades of life (reported mean age of 14 years) (4). The high propensity of local recurrence (20-30\%) is reported (5). This tumor is composed of cells with myofibroblastic differentiation, prominent loose bundle of fibrous tissue and collagen fibers, absence of mitoses or atypical cells $(3,5,6)$. Intraosseous fibroma is divided into two subgroups of odontogenic fibroma and non-odontogenic fibroma (desmoplastic fibroma). Although the clinical findings of both are similar, non-odontogenic type is more aggressive (7). This tumor mostly occurs in mandible, femur, humerus and pelvis $(2,3,5,6,8)$. However, it is believed that DF can arise in any bone of the body $(2,3,9,10)$. From the epidemiologic point of view, the presence of DF instead of desmoplastic fibroma in long and flat bones is similar $(2,7,10)$. The incidence of DF in zygoma is extremely rare and only three cases have been reported till now. In 1958, DF was firstly described by Jaffe as an uncommon benign tumor in the skeleton, which involved multiple sites of tibia, scapula and femur (11). In 1965, the first case of gnathic involvement with DF was introduced by Griffith and Irby (12). High recurrence rate of this lesion makes en bloc resection the treatment of choice $(2,3,13,14)$.

\section{Case Presentation}

A 6-year-old girl was referred to a dentomaxillofacial private radiology clinic for professional consultation regarding a mass over the right cheek region with chief complaints of pain and swelling growing for less than 2-3 weeks. The patient underwent clinical examination. Her medical history was unremarkable. Patient was not taking any medication. There was no evidence of lymphadenopathy. The extraoral examination showed a single diffuse swelling along the right infraorbital rim, within the right zygomatic bone (Figure 1). On palpation, the mass was found to be bony hard, nonfluctuant and nontender. No paresthesia was reported. Overlying skin was normal. Intraoral examination was within normal limits. Conebeam computed tomography (CBCT) was ordered to evaluate the swelling. The patient was scanned by SCANORA 3D CBCT unit (SOREDEX, Helsinki, Finland) with a $7.5 \times$ $10 \mathrm{~cm}$ FOV (Field of View) and $200 \mu \mathrm{m}$ voxel size. CBCT revealed a mixed lesion in infraorbital rim, which had ill-defined borders with the presence of a straight thick bony septum in the lesion. It also involved the zygomatic process of maxilla and zygomatic bone. The expansion of this destructive lesion was $13 \mathrm{~mm}$ (anteroposteriorly), $16 \mathrm{~mm}$ (mediolaterally) and $12 \mathrm{~mm}$ (superoinferiorly). A region of perforation and breaking through outer cortex and extension into the overlying soft tissue was ob-

Copyright (C) 2015, Tehran University of Medical Sciences and Iranian Society of Radiology. This is an open-access article distributed under the terms of the Creative Commons Attribution-NonCommercial 4.0 International License (http://creativecommons.org/licenses/by-nc/4.0/) which permits copy and redistribute the material just in noncommercial usages, provided the original work is properly cited. 
served without any periosteal reaction (Figures 2 - 4 ). The patient underwent surgery under general anesthesia via an intraoral approach. The lesion was removed by En bloc resection containing the inferior orbital rim, zygomatic buttress and partial of orbital floor, while the Infraorbital nerve was preserved. At the same time, reconstruction of defect was performed by alloplast (Porex Surgical Inc., College Park, GA, USA). The postoperative specimen was re-evaluated microscopically. The histopathological examination revealed that the tumoral lesion consisted of varying degrees of spindle cells, proliferation and round to ovoid cells in some areas arranged in interlacing bundles and fascicles. Bony spicules present at the interface between the tumor and adjacent bone (Figure 5). Based on imaging and histopathologic findings, a diagnosis of DF was made.

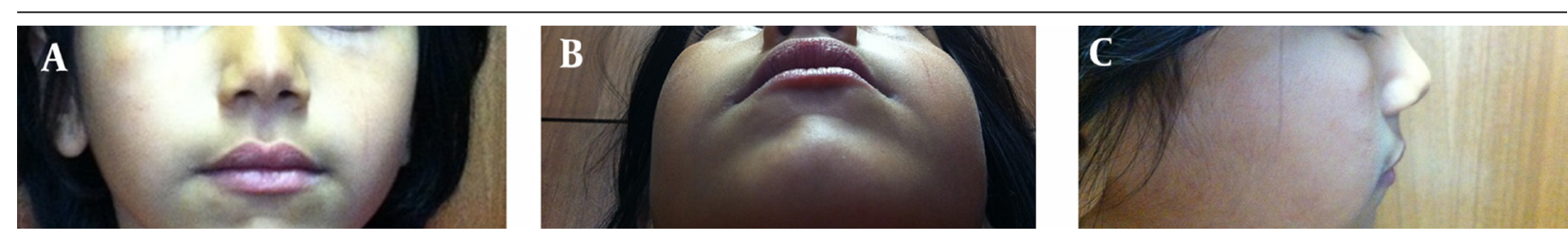

Figure 1. A 6-year-old girl with pain and swelling over the right cheek region for less than 2-3 weeks. A, Frontal; B, submental and C, right lateral views of the patient. Note the slightly swelling of the right infraorbital region.
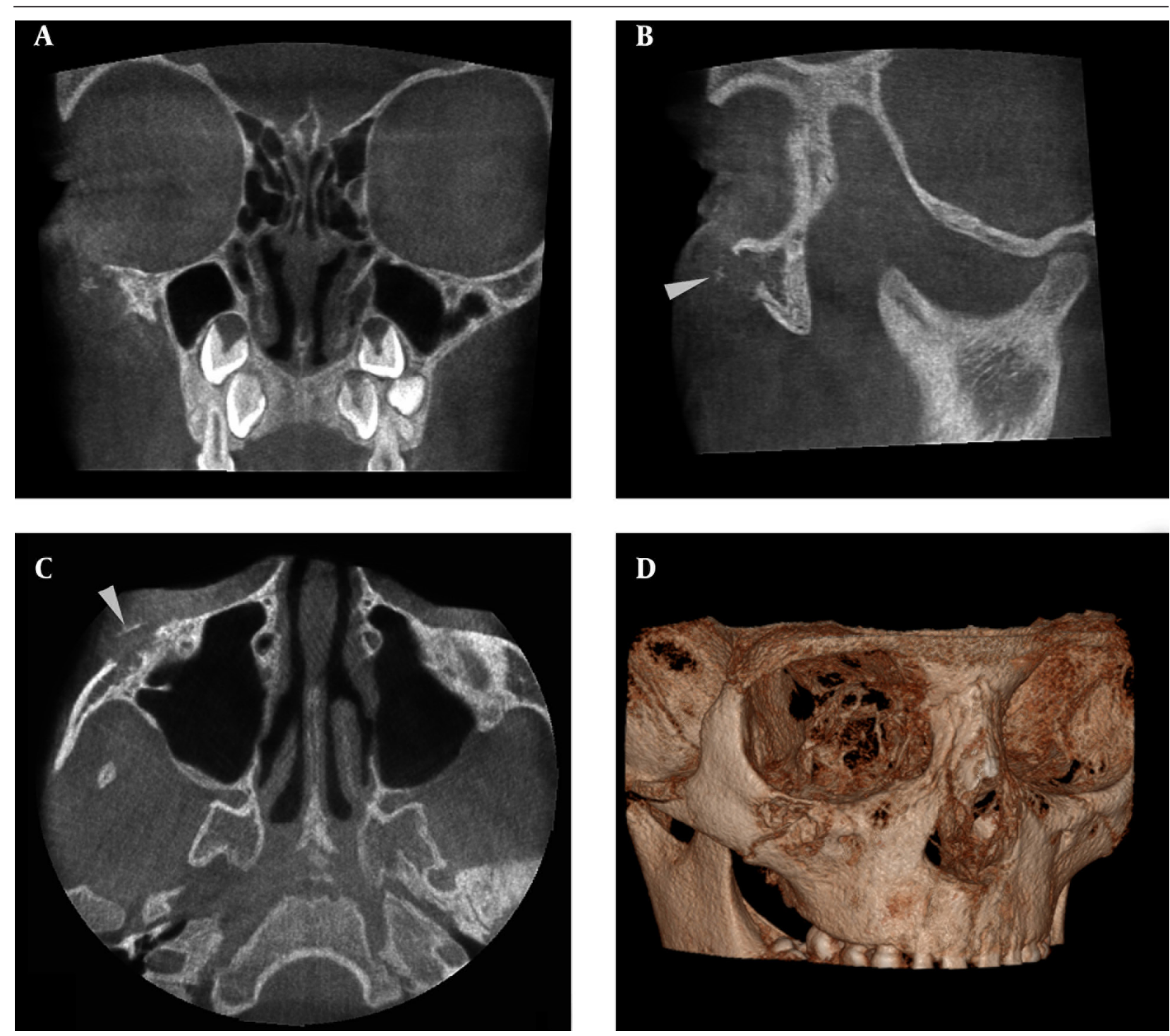

Figure 2. A, Coronal; B, Sagittal; C, Axial and D, 3D views of cone beam computed tomography demonstrate a lytic lesion in the right infraorbital rim and zygomatic buttress. note the presence of a coarse straight septum (arrowhead). 

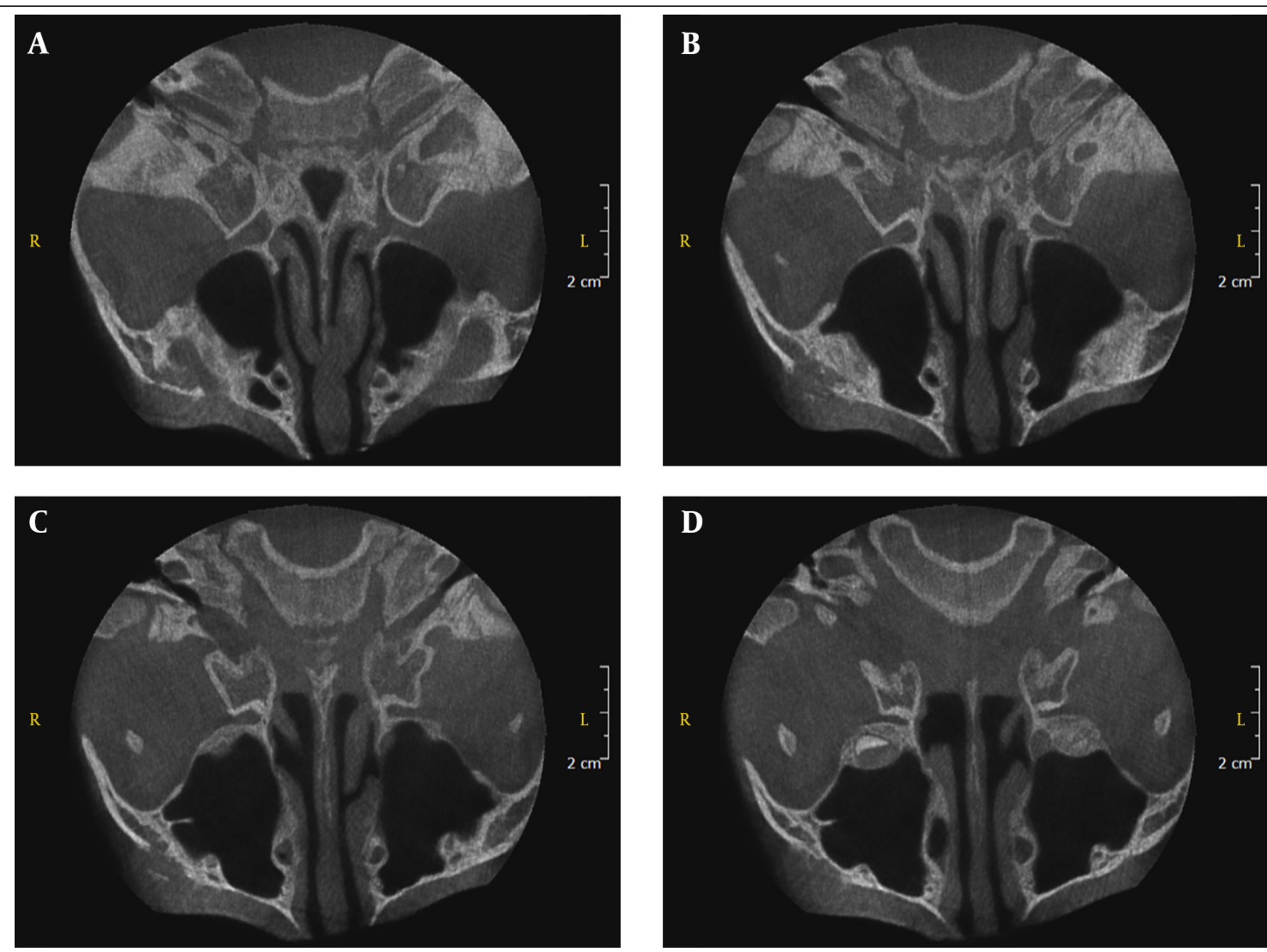

Figure 3. Series of axial Cone Beam Computed Tomography view demonstrate a mixed lesion in the right zygomatic buttress.
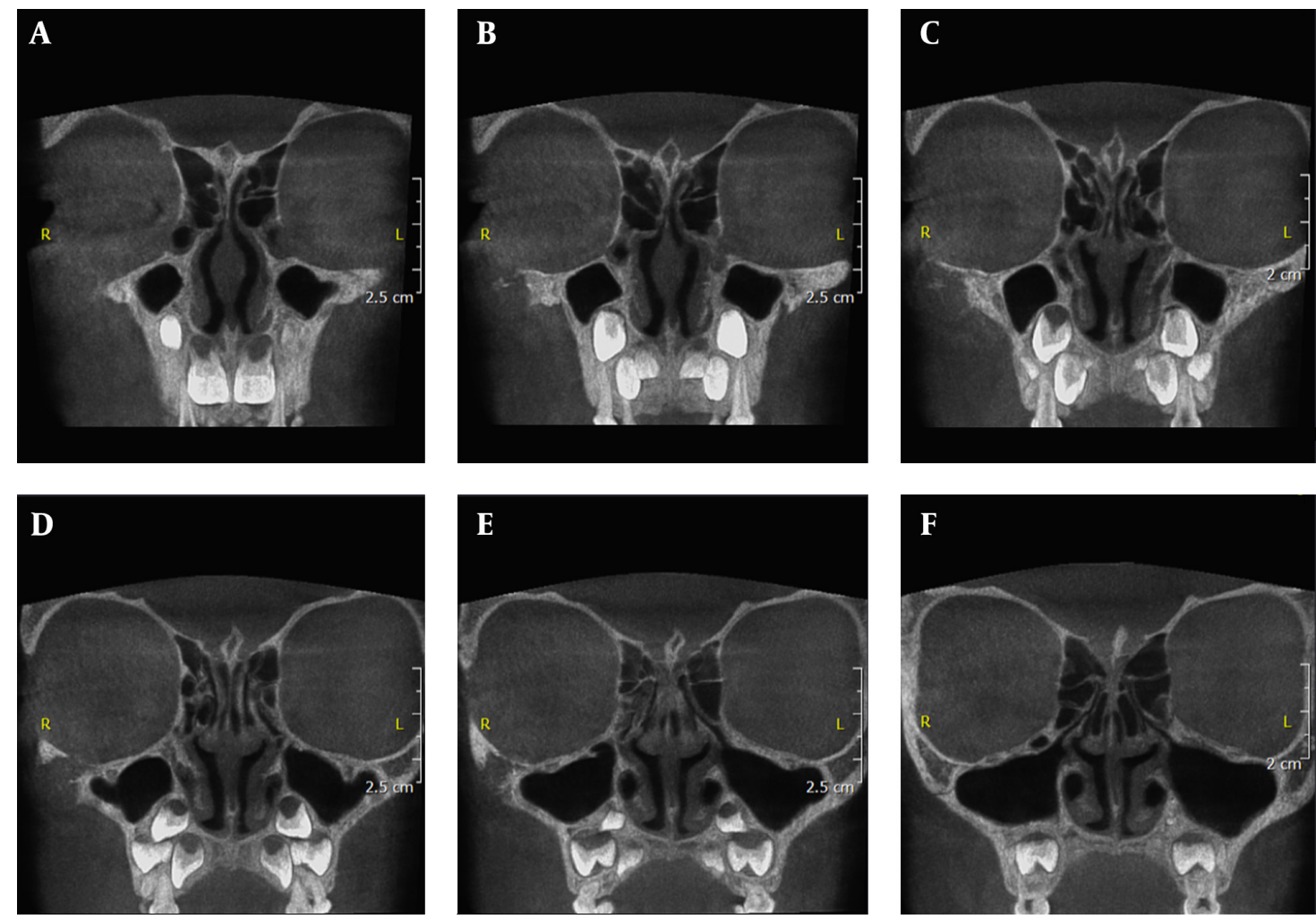

Figure 4. Series of coronal Cone Beam Computed Tomography view demonstrate a lytic lesion in the right infraorbital rim and zygomatic buttress. 


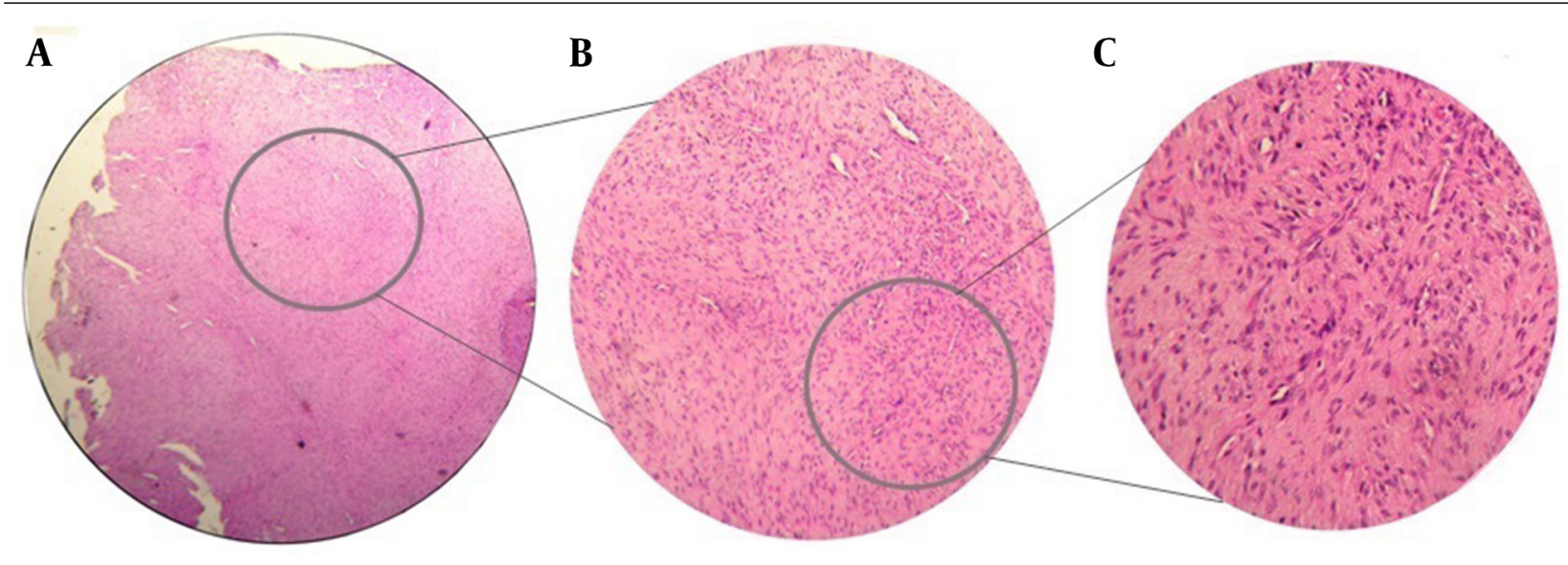

Figure 5. Low-power photomicrographs show spindle cell, proliferation and round to ovoid cells (hematoxylin-eosin stain). A, original magnification $\times 40$. B, original magnification $\times 100$. C, original magnification $\times 400$.

\section{Discussion}

DF is a locally aggressive lytic benign tumor of bone with a tendency to involve children (7). The incidence of DF is far less than $0.1 \%$ among all benign bone tumors $(1,3,9,14)$. The etiology of DF is still unknown (3, $6)$. There are some hints of possible endocrine, genetic factors and trauma (2), but none of them is proved as the etiologic agent. A wide age distribution has been reported for DF occurrence, extending from birth to the sixth decade of life, with a peak incidence at 10 to 19 years of age. Some literature reviews represent a slight difference in gender distribution, while others believe equal occurrence in both genders (2, 6, 8, 9). Generally, there is no pathognomonic sign and symptom for DF $(6,8)$, but pain and swelling have been mentioned by most patients $(1-3,13,14)$. Fractures are more common in long bones $(2,3)$. Any bone could be involved with DF $(2,3,10)$. Among facial bones, the mandible is the most common site of involvement $(2,6-8,10)$. This appears to be the fourth case report of such a diagnosis in zygomatic bone (Table 1). Radiographically, DF appearance is nonspecific and usually mimics other lytic lesions $(2,13)$. These lesions are characterized by bone expansion, well or ill-defined margins, benign features with pattern of destruction, multi- or unilocular and totally radiolucent or radiolucent-radiopaque lesions (15). The multilocularity is three times more likely based on previous reports (7), which could be described by different locations. The presence of a coarse, irregular or straight septum is possible in this kind of lesion (15), which was identical in our case (Figure $2 \mathrm{C}$ ). Based on previous case reports, destruction and perforation of cortex are characteristic features of DF (7). Presence of reactive sclerosis at the tumor margins was mentioned in the case report of Smith et al. (2), while it was undetected in our case. Imaging features revealed mixed lesion without periosteal reaction in Jamali et al. (3), which is compatible with our finding in the recent case. Jamali et al. (3) and

\begin{tabular}{lccc}
\hline \multicolumn{3}{l}{ Table 1. List of Reports of DF in Zygomatic Bone } & \\
\hline Author (s) & $\begin{array}{c}\text { Year of } \\
\text { Report }\end{array}$ & $\begin{array}{c}\text { Age of } \\
\text { Patient (s) }\end{array}$ & Gender \\
\hline Jamali et al. (3) & 2013 & 38 & Male \\
Smith et al. (2) & 1999 & 5 & Male \\
Bontemps et al.(13) & 2014 & 26 & Female \\
Present study & 2015 & 6 & Female \\
\hline
\end{tabular}

Smith et al. (2) reported that DF involved the lateral wall of orbit, also with extending approximately to the base of skull, whereas in the present case, the lateral wall of orbit was intact. DF occupied the zygomatic process of maxilla, zygomatic bone, infraorbital rim and orbit floor. CBCT images also revealed perforation and breaking through outer cortex and extension into the overlying soft tissue. DF is an aggressive tumor with high recurrence rate, so it is important to distinguish it from other lesions with similar characteristics. The differential diagnosis includes myxoma, giant cell tumors, aneurysmal and solitary bone cyst, hemangioma, fibrous dysplasia, chondromyxoid fibroma, ossifying fibroma, sarcomas and metastatic lesions $(6,10)$. It is believed that diagnosis of DF is possible only by histopathologic examination (3). Different treatment methods have been suggested for DF that are the source of controversy (8). Enucleation, curettage, resection, radiotherapy (10) and chemotherapy are usual implemented treatment modalities. Radiotherapy due to its mutagenic effect is not recommended in most literatures $(2,3,5$, $6,13)$. This method is likely to cause postradiation sarcoma $(2,3,13)$. Studies do not support that lesions with increased cellularity have a higher tendency to recur. Some reports indicate that curettage has a greater risk of recurrence $(1,3,6)$. En bloc resection is the treatment 
of choice $(2,3,13,14)$. According to radiologic concepts, this rare lesion may mimic fibro-osseous, benign and especially malignant lesions. Regarding different treatment plans, identification of this lesion is essential. Additionally, the presence of coarse and irregular or straight septa along with some imaging criteria for malignant lesions such as destruction of the cortex, periosteal reaction and soft tissue invasion would be helpful to differentiate this lesion from malignant and multilocular benign lesions.

\section{Acknowledgements}

I would like to offer my gratitude to my dear colleagues in Maxillofacial Radiology, Pathology and Surgery Departments who have assisted me in preparing this case report.

\section{Authors' Contributions}

Study concept and design: Yaser Safi, Solmaz Valizadeh and Maryam Eslami Manouchehri. Analysis and interpretation: Yaser Safi, Nafise Shamloo, Hossein Heidar, Solmaz Valizadeh, Mohammad Mehdi Aghdasi and Maryam Eslami Manouchehri. Critical revision of the manuscript for important intellectual content: Yaser Safi, Nafise Shamloo, Hossein Heidar, Solmaz Valizadeh, Mohammad Mehdi Aghdasi and Maryam Eslami Manouchehri. Drafting of the manuscript: Yaser Safi, Solmaz Valizadeh and Maryam Eslami Manouchehri. Study supervision: Yaser Safi, Nafise Shamloo, Hossein Heidar, Solmaz Valizadeh, Mohammad Mehdi Aghdasi and Maryam Eslami Manouchehri.

\section{Financial Disclosure}

We had no financial interests related to the material in the manuscript.

\section{Funding/Support}

We received no funding related to this manuscript.

\section{References}

1. Schneider M, Zimmermann AC, Depprich RA, Kubler NR, Engers $\mathrm{R}$, Naujoks CD, et al. Desmoplastic fibroma of the mandible--review of the literature and presentation of a rare case. Head Face Med. 2009;5:25.

2. Smith ML, Williams JK, Gruss JS. Desmoplastic fibroma of the zygoma. Plast Reconstr Surg. 1999;103(7):1964-9.

3. Jamali M, Lazow SK, Solomon MP, Berger J. Desmoplastic fibroma: report of rare lesion in unusual craniofacial location. $N$ Y State Dent J. 2013;79(3):43-5.

4. Takazawa K, Tsuchiya H, Yamamoto N, Nonomura A, Suzuki M, Taki J, et al. Osteosarcoma arising from desmoplastic fibroma treated 16 years earlier: a case report. J Orthop Sci. 2003;8(6):864-8.

5. Daneyemez M, Akay KM, Izci Y. Desmoplastic fibroma of the cervical spine. Eur Spine J. 2005;14(8):799-802.

6. Said-Al-Naief N, Fernandes R, Louis P, Bell W, Siegal GP. Desmoplastic fibroma of the jaw: a case report and review of literature. Oral Surg Oral Med Oral Pathol Oral Radiol Endod. 2006;101(1):82-94.

7. Ikeshima A, Utsunomiya T. Case report of intra-osseous fibroma: a study on odontogenic and desmoplastic fibromas with a review of the literature. J Oral Sci. 2005;47(3):149-57.

8. Stefanidis K, Benakis S, Tsatalou E, Ouranos V, Chondros D. Computed tomography and magnetic resonance imaging of desmoplastic fibroma with simultaneous manifestation in two unusual locations: a case report.J Med Case Rep. 2011;5:28.

9. Vanhoenacker FM, Hauben E, De Beuckeleer LH, Willemen D, Van Marck E, De Schepper AM. Desmoplastic fibroma of bone: MRI features. Skeletal Radiol. 2000;29(3):171-5.

10. Sandrini FA, do Egito Vasconcelos BC, Bessa-Nogueira RV. Desmoplastic fibroma of mandible in a child: case report. Indian J Surg. 2007;69(6):254-6.

11. Jaffe H. Tumors and tumorous conditions of the bones and joints. Philadelphia: Lea \& Febiger; 1958. pp. 298-303.

12. Griffith JG, Irby WB. Desmoplastic Fibroma. Report of a Rare Tumor of the Oral Structures. Oral Surg Oral Med Oral Pathol. 1965;20:269-75.

13. Bontemps P, Brix M, Simon E. Fibrome desmoplastique de l'os zygomatique. Rev Stomatol Chir Maxillofac Chir Orale. 2014;115(3):169-71.

14. Taconis WK, Schutte HE, van der Heul RO. Desmoplastic fibroma of bone: a report of 18 cases. Skeletal Radiol. 1994;23(4):283-8.

15. White SC, Pharoah MJ.Oral radiology: principles and interpretation. Elsevier Health Sciences; 2013. 